\title{
Electrostatic and (electro-)steric stabilization of colloidal particles by natural organic matter
}

\section{SENIHA SIMALE SU UYGAN AND STEFAN SCHYMURA}

Helmholtz-Zentrum Dresden - Rossendorf e. V. (HZDR)

Presenting Author: s.uygan@hzdr.de

Natural organic matter, such as humic and fulvic acids play a major role in the mobility of contaminants in the environment [1] including radionuclides and nanoparticles formed by them [2]. Humic and fulvic acids may act as stabilizers, i.e. natural surfactants, for colloids, thereby increasing their stability and mobility in the Geo- and Biosphere. This stabilization of colloidal matter is caused by three basic mechanisms: (1) electrostatic stabilization, (2) electrosteric stabilization and (3) steric stabilization [3]. These mechanisms can contribute to different degrees to the stabilization of particulate contaminants by natural organic matter in colloidal dispersion and also influence their interactions with mineral surfaces. In order to understand the different contributions controlled homoaggregation studies on monodisperse nanoparticles have been performed using model stabilizers, such as ionic surfactants and polymers. These model scenarios allowed us to isolate electrostatic and steric components in aggregation studies using dynamic light scattering. By comparing these results to systems with different humic and fulvic acids we can elucidate which components control the stabilization of colloidal contaminants by natural organic matter.

[1] Aiken, Hsu-Kim \& Ryan (2011) Environ. Sci. Technol. 45, 8, 3196-3201

[2] Stockdale \& Bryan (2013) Earth Science Reviews 121, 117

[3] Tiller \& O’Melia (1993), Colloids and Surfaces A 73, 89102. 